\section{Protection from lonizing Radiation}

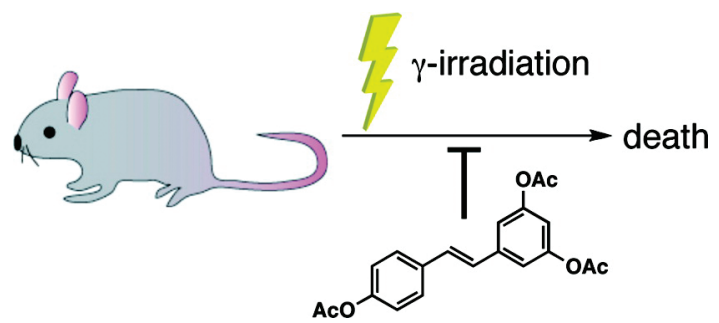

Exposure to ionizing radiation can result in morbidity and even death. As recent catastrophic events in the wake of an earthquake and tsunami in Japan demonstrate, the need for radioprotective agents is of medical importance. Currently, efforts are underway to discover novel radioprotective agents that can limit the deleterious effects of acute exposure to high quantities or prolonged exposure to lower quantities of ionizing radiation. It is known that reactive oxygen species are produced on exposure to radiation. On the basis of this observation, Koide et al. (DOI: 10.1021/ml100159p) searched among natural polyphenol antioxidants with the assumption that some of these might serve as good starting lead compounds for clinical radioprotective agents.

The authors focused on resveratrol, a natural product found, among other foods, in grapes, nuts, and wine. Because resveratrol has poor bioavailability and pharmacokinetic properties, the authors synthesized a prodrug with similar structural properties but with three acetyl groups. When this compound was administered with an excipient to mice exposed to radiation, it resulted in increased survival.

\section{Antibiotics That Bind to Bacterial DNA Gyrase}

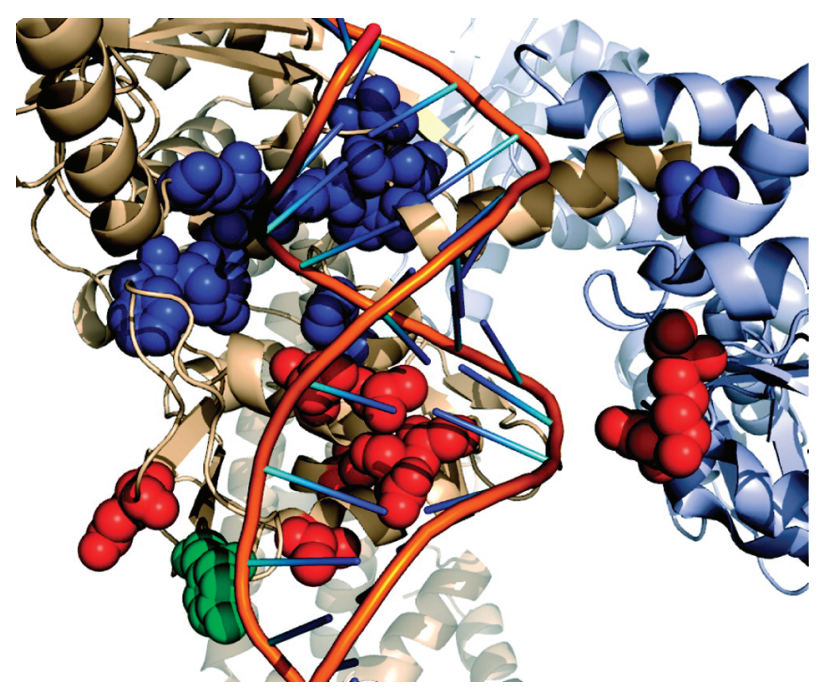

DNA gyrase is an enzyme that catalyzes the ATP-dependent introduction of negative supercoils in DNA. Because it is an essential enzyme in bacteria, it is an important target in the development of new antibiotics. Foss et al. (DOI: 10.1021/ ml1002822) describe a new class of inhibitors of bacterial DNA gyrase.

Gyramide A had previously been demonstrated to exhibit antibacterial activity. In the current study, the authors sequenced the genome of bacteria, which had developed resistance to this antibiotic, and found that they possessed mutations that mapped to the gene encoding DNA gyrase. Furthermore, the authors found that these small molecules were effective against certain Gram-negative and Gram-positive bacteria when combined with an efflux pump inhibitor.

\section{A MEK Inhibitor for Cancer Treatment}<smiles>CC(=O)Nc1cccc(-n2c(=O)n(C3CC3)c(=O)c3c(Nc4ccc(I)cc4F)n(C)c(=O)c(C)c32)c1</smiles>

The mitogen-activated protein kinase/extracellular signalregulated kinase kinase (MEK) is associated with a number of malignancies. Inhibition of MEK is therefore a valid target in cancer drug discovery. Abe et al. (DOI: $10.1021 / \mathrm{ml} 200004 \mathrm{~g}$ ) identified a lead compound from a cell-based high-throughput screen. The selectivity and potency of inhibitors were improved by structure-activity relationship analysis.

As a result of these efforts, the authors developed a first-inclass MEK inhibitor with a unique chemical core. The antiproliferative activity of this inhibitor was tested in cellular assays and in a tumor xenograft model. The compound described in this study is currently in phase III clinical tries for certain types of melanomas. 